\title{
ACCRETION OF A TERRESTRIAL-LIKE MINOR PLANET BY A WHITE DWARF
}

\author{
Carl Melis $^{1,6}$, J. Farihi ${ }^{2}$, P. Dufour ${ }^{3}$, B. Zuckerman ${ }^{4}$, Adam J. Burgasser $^{1,5}$, P. Bergeron ${ }^{3}$, J. Bochanski $^{5}$, And R. Simcoe ${ }^{5}$ \\ ${ }^{1}$ Center for Astrophysics and Space Sciences, University of California, San Diego, CA 92093-0424, USA; cmelis@ ucsd.edu \\ 2 Department of Physics \& Astronomy, University of Leicester, Leicester LE1 7RH, UK \\ ${ }^{3}$ Département de Physique, Université de Montréal, Montréal, QC H3C 3J7, Canada \\ ${ }^{4}$ Department of Physics and Astronomy, University of California, Los Angeles, CA 90095-1547, USA \\ ${ }^{5}$ Massachusetts Institute of Technology, Kavli Institute for Astrophysics and Space Research, Building 37, Room 664B, 77 Massachusetts Avenue, \\ Cambridge, MA 02139, USA \\ Received 2010 November 22; accepted 2011 February 1; published 2011 April 21
}

\begin{abstract}
We present optical and infrared characterization of the polluted DAZ white dwarf GALEX J193156.8+011745. Imaging and spectroscopy from the ultraviolet to the thermal infrared indicate that this white dwarf hosts excess infrared emission consistent with the presence of an orbiting dusty debris disk. In addition to the five elements previously identified, our optical echelle spectroscopy reveals chromium and manganese and enables restrictive upper limits on several other elements. Synthesis of all detections and upper limits suggests that GALEX J193156.8+011745 has accreted a differentiated parent body. We compare the inferred bulk elemental composition of the accreted parent body to expectations for the bulk composition of an Earth-like planet stripped of its crust and mantle and find relatively good agreement. At least two processes could be important in shaping the final bulk elemental composition of rocky bodies during the late phases of stellar evolution: irradiation and interaction with the dense stellar wind.
\end{abstract}

Key words: circumstellar matter - planet-star interactions - stars: abundances - stars: individual (GALEX

J193156.8+011745) - white dwarfs

Online-only material: color figures

\section{INTRODUCTION}

Recent studies have solidified the paradigm that metal-rich white dwarfs are polluted by remnant rocky bodies from their planetary systems (e.g., Zuckerman et al. 2007; Jura 2008; Farihi et al. 2009, 2010a, 2010b; Dufour et al. 2010; Klein et al. 2010; Melis et al. 2010; Zuckerman et al. 2010; and references therein). It is now desirable to understand the mass, nature, and history of the accreted parent body at each white dwarf. The larger the degree of pollution, the greater the potential for insight into extrasolar rocky bodies.

Vennes et al. (2010) describe a heavily polluted DAZ white dwarf-GALEX J193156.8+011745 (hereafter GALEX1931) exhibiting atmospheric magnesium, silicon, oxygen, calcium, and iron $(\mathrm{Mg}, \mathrm{Si}, \mathrm{O}, \mathrm{Ca}$, and $\mathrm{Fe}$, respectively). Additionally, Two Micron All Sky Survey (2MASS; Skrutskie et al. 2006) photometry suggests excess near-infrared emission. Abundance analysis and the shape of the putative infrared excess were interpreted by Vennes et al. (2010) as consistent with a midL-type dwarf that orbits and pollutes GALEX1931. However, near-infrared emission from a prominent dust disk is also consistent with the spectral energy distribution presented by Vennes et al. (2010). Debes et al. (2011) report Widefield Infrared Survey Explorer (WISE) imaging and optical spectroscopic monitoring of GALEX1931 that support a disk interpretation.

Here we present optical through infrared imaging and spectroscopy of GALEX1931 designed to better constrain the presence of infrared excess emission and to probe the material polluting the white dwarf star's atmosphere.

\footnotetext{
6 Joint CASS Departmental Fellow and NSF AAPF Fellow.
}

\section{OBSERVATIONS}

\subsection{Nickel Optical Imaging}

Optical imaging was performed on UT 2010 May 25 at Lick Observatory with the 40 inch Nickel telescope. These observations used the facility's Direct Imaging Camera (CCDC2), a $2048 \times 2048$ pixel detector with $15 \mu$ m pixels. The 0'. 184 pixel $^{-1}$ plate scale affords a field of view of $\sim 6 ! 3$ squared. The detector was binned by two in rows and columns and was read out in fast mode. Occasional clouds passed through the region of the sky being observed; frames affected by clouds were not used in the final analysis.

GALEX1931 was observed in each of the BVRI filters (Bessell 1990). For each filter, a four-step dither pattern with 10" steps was repeated with $20 \mathrm{~s}$ integrations per step position. The flux calibrator star SA 111-1925 (Landolt 2009) was visited following the completion of a dither sequence for each filter. Images are reduced by median combining all good frames to obtain a "sky" frame and subtracting this sky frame from each image. Sky-subtracted images are then divided by flatfield frames obtained by imaging the twilight sky $(B V R)$ or the illuminated telescope dome $(I)$. Each science frame is registered using bright stars in the field and then all science frames are median combined to yield the final reduced image. Detector counts for GALEX1931 and SA 111-1925 are extracted with an aperture that yields $\sim 85 \%$ encircled energy (with a negligible correction between the two stars). This is achieved by extracting counts for both stars with a 6 pixel (2".2) radius circular aperture. Sky is sampled with an annulus extending from 20 to 100 pixels. Uncertainties are derived from the dispersion of measurements made from individually reduced frames. The uncertainties for SA 111-1925 are propagated into the final quoted uncertainty 

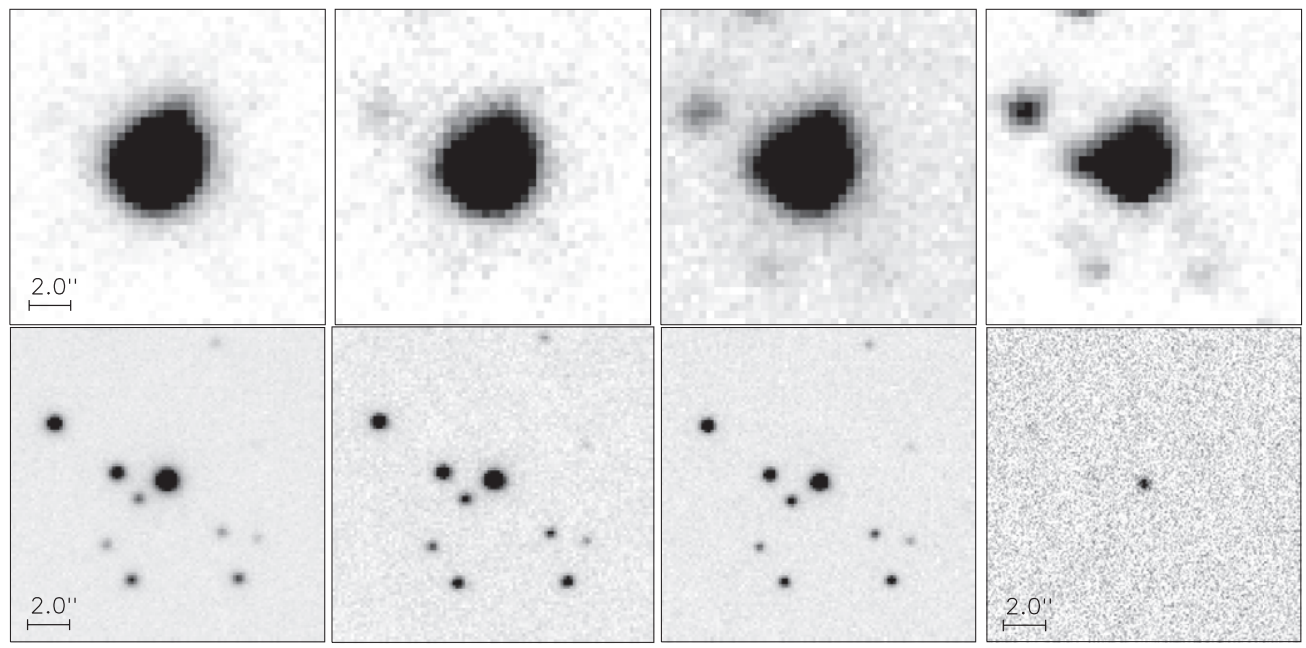

Figure 1. Images of GALEX1931 (center star in each frame). North is up and east is left in each image. Top row: $B V R I$ (from left to right) images from the Nickel $1 \mathrm{~m}$. All images are presented with the same inverted linear stretch. Bottom row: $J_{s} H K_{s} L^{\prime}$ (from left to right) images from ISAAC. The $J_{s} H K_{s}$ images are all presented with the same inverted linear stretch. Note that the two objects within $\approx 2^{\prime \prime}$ of GALEX1931 are red.

Table 1

Broadband Fluxes for GALEX1931

\begin{tabular}{lccc}
\hline \hline Band & $\begin{array}{c}\lambda \\
(\mathrm{nm})\end{array}$ & $\begin{array}{c}m^{\mathrm{a}} \\
(\mathrm{mag})\end{array}$ & $\begin{array}{c}F_{\text {obs }} \\
(\mathrm{mJy})\end{array}$ \\
\hline$L^{\prime}$ & 3780 & $14.00 \pm 0.14$ & $0.63 \pm 0.08^{\mathrm{b}}$ \\
$K_{s}$ & 2160 & $14.62 \pm 0.05$ & $0.95 \pm 0.05$ \\
$H$ & 1650 & $14.80 \pm 0.05$ & $1.26 \pm 0.06$ \\
$J_{S}$ & 1240 & $14.80 \pm 0.05$ & $1.96 \pm 0.10$ \\
\hline \multicolumn{3}{c}{ Nickel $^{\mathrm{a}}-$ Optical } \\
\hline & 798.2 & $14.47 \pm 0.04$ & $3.94 \pm 0.16$ \\
$R$ & 640.7 & $14.31 \pm 0.03$ & $5.79 \pm 0.17$ \\
$V$ & 544.8 & $14.20 \pm 0.02^{\mathrm{c}}$ & $7.52 \pm 0.15$ \\
$B$ & 436.3 & $14.18 \pm 0.02$ & $8.55 \pm 0.17$ \\
\hline \multicolumn{3}{c}{$G A L E X^{\mathrm{a}}-$ Ultraviolet } \\
\hline NUV & 227.1 & $13.70 \pm 0.03$ & $11.94 \pm 0.36$ \\
FUV & 152.8 & $13.46 \pm 0.05$ & $14.99 \pm 0.75$ \\
\hline
\end{tabular}

Notes.

a ISAAC and Nickel magnitudes are on the Vega system. GALEX measurements are in $\mathrm{AB}$ magnitudes and have uncertainties as suggested in Morrissey et al. (2007).

b This value disagrees with the WISE $3.35 \mu \mathrm{m}$ measured value of $1.02 \pm$ 0.04 mJy (Debes et al. 2011); see Section 3.2.

c This measurement is from Vennes et al. (2010); see Section 2.1.

for GALEX1931. The final $V$-band measurement from the Nickel observations is less accurate than that reported in Vennes et al. (2010); we thus adopt the Vennes et al. (2010) $V$-band measurement.

Nickel images are presented in Figure 1 and photometry is reported in Table 1 which also includes Galaxy Evolution Explorer (GALEX; Martin et al. 2005) fluxes.

\subsection{ISAAC Near-infrared Imaging}

Near-infrared imaging was performed with ISAAC (Moorwood et al. 1998) mounted on the VLT-UT3 telescope. Data were obtained on UT 2010 October 29 at Cerro Paranal in excellent conditions, resulting in stellar images with FWHM $<0$ '. 5 in the $J_{s}$ and $H$ bands and $<0^{\prime \prime} .4$ at $K_{s}$. The $J_{s}$ filter was employed due to a red ( $K$ band) leak in the standard $J$ filter.
Utilizing individual exposure times of $10 \mathrm{~s}$, a nine-point dither pattern with non-uniformly spaced positions inside a $30^{\prime \prime}$ bounding box was executed at each of $J_{s} H K_{s}$. The standard star EG 141 (Hawarden et al. 2001) was observed in an identical manner but with $5 \mathrm{~s}$ individual exposures.

These data were reduced at the telescope by the Gasgano ISAAC pipeline. A sky frame is generated from the median of all the science images and subtracted from individual exposures. A master flat field is employed to correct pixel-to-pixel gain variation. Individual science frames are registered using twodimensional cross-correlation and averaged to yield a fully reduced image. Photometry is performed on both GALEX1931 and the standard star with the IRAF task apphot using an 8 pixel (1".2) aperture radius and a 20 to 30 pixel sky annulus. Flux measurements for GALEX1931 are corrected for a mild airmass difference between GALEX1931 and EG 141 that amounts to $<0.02 \mathrm{mag}$, and flux-calibrated on the Vega system. Although $\mathrm{S} / \mathrm{N} \gtrsim 300$ was achieved for the science target at all three bandpasses, a conservative error of $5 \%$ is chosen for the derived magnitudes to compensate for uncharacterized filter transformation functions for the ISAAC filters and other uncharacterized systematics (see e.g., Leggett et al. 2006).

Imaging photometry of GALEX1931 was also performed at $L^{\prime}$ using both telescope nodding and secondary mirror chopping. An ABBA nodding pattern was repeated with several arcseconds between each nod position and approximately $30 \mathrm{~s}$ spent at each nod step (or $120 \mathrm{~s}$ for the entire cycle). At each nod position, chop throws of $20^{\prime \prime}$ were utilized at a frequency of $0.43 \mathrm{~Hz}$ with individual exposure times of $0.11 \mathrm{~s}$ and nine co-adds at each mirror position. Additionally, the position of the ABBA pattern on the array was non-uniformly dithered between cycles within a $10^{\prime \prime}$ box. The total on-source time for GALEX1931 was $1188 \mathrm{~s}$, consisting of 30 chopping cycles nested within 10 nodding cycles of four positions each. The $L^{\prime}$ standard star FS 148 (Leggett et al. 2003) was observed similarly with a total on-source time of $118.8 \mathrm{~s}$ over one complete nodding cycle.

A combined and reduced $L^{\prime}$ frame was generated at the telescope by the pipeline. The two co-added and chop-subtracted images produced at each consecutive pair of nod positions are combined, and then divided by a master flat field. Image alignment is achieved by compensating for the executed telescope offsets between nodding, and the two images are averaged. 
Table 2

Spectroscopic Observations Summary

\begin{tabular}{lcccccrr}
\hline \hline UT Date & Instrument & Setup & Coverage & Resolution $^{\mathrm{a}}$ & Integration Time $(\mathrm{s})^{\mathrm{S} / \mathrm{N}}$ & $\lambda$ of $\mathrm{S} / \mathrm{N}^{\mathrm{b}}(\AA)$ \\
\hline 2010 Jul 5 & HIRES & UV collimator & $3130-5940 \AA$ & $\sim 40,000$ & $2 \times 1800$ & $80^{\mathrm{c}}$ & 3450 \\
2010 Sep 19 & FIRE & Prism mode & $0.7-2.5 \mu \mathrm{m}$ & $\approx 250-350^{\mathrm{d}}$ & $4 \times 60$ & $80^{\mathrm{c}}$ & 10500 \\
2010 Oct 9 & KAST & $600 / 4310$ & $3430-5510 \AA$ & $\sim 3.4 \AA$ & 600 & 100 & 4500 \\
& & $1200 / 5000$ & $5520-6910 \AA$ & $\sim 2.4 \AA$ & 600 & 60 & 6300 \\
\hline
\end{tabular}

Notes.

a Resolutions were measured from the FWHM of single arclines in our comparison spectra.

b Wavelength where $\mathrm{S} / \mathrm{N}$ measurement was made in the spectrum.

${ }^{c} \mathrm{~S} / \mathrm{N}$ for combined exposures.

${ }^{\mathrm{d}}$ See Burgasser et al. (2010).

e Observations used the 2 .'0 slit and the d55 dichroic.

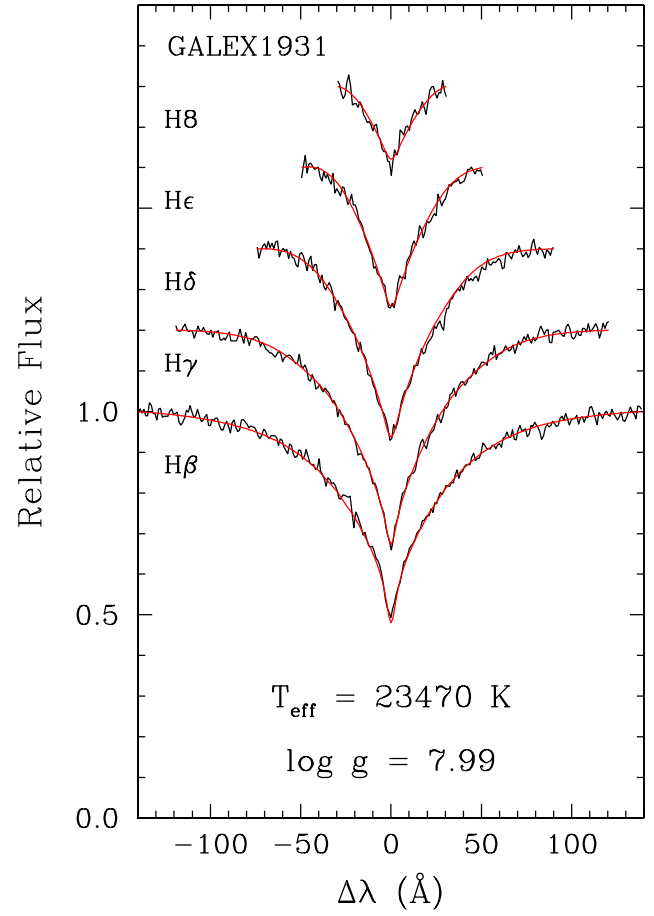

Figure 2. Best-fit models (smooth red lines) to Balmer lines in the KAST blue side data of GALEX1931 (underlying black spectrum). The best-fit $T_{\text {eff }}$ and $\log g$ are also displayed.

(A color version of this figure is available in the online journal.)

The difference in chopped images effectively removes the sky background, and the observing pattern results in one positive and two negative stellar images in the recombined frame. A final, reduced image is constructed by combining (aligning and averaging) all the frames obtained in this manner. Photometry on both GALEX1931 and the standard star is performed with apphot using an aperture radius of 20 pixels (1".4) and a sky annulus of 30 to 50 pixels. The difference in airmass between GALEX1931 and FS 148 implies an extinction correction of less than $0.01 \mathrm{mag}$, and the flux of GALEX1931 is calibrated on the Vega system. A total photometric error is calculated by the quadrature sum of an (assumed) 5\% calibration uncertainty and the measured $\mathrm{S} / \mathrm{N}=9.2$ at the photometric aperture radius.

ISAAC photometric results are listed in Table 1. Figure 1 shows the ISAAC images of GALEX1931. It is noted that the $L^{\prime}$-band flux detected from this imaging differs significantly from the $3.35 \mu \mathrm{m}$ flux detected toward GALEX1931 by the WISE satellite of $1.02 \pm 0.04 \mathrm{mJy}$ (Debes et al. 2011). Possible reasons for this discrepancy are discussed in Section 3.2.

\subsection{Optical Spectroscopy}

Grating resolution optical spectroscopy was performed at Lick Observatory with the KAST Double Spectrograph mounted on the Shane $3 \mathrm{~m}$ telescope. Table 2 lists the observation date and instrumental setup. Data are reduced using standard IRAF long-slit tasks. Absolute flux calibration of the KAST data is accomplished by scaling to the Nickel $B$ - and $R$ band measurements and the Vennes et al. (2010) $V$-band measurement. KAST data are shown in Figures 2 and 3.

Keck High Resolution Echelle Spectrometer (HIRES; Vogt et al. 1994) optical echelle spectra were obtained for GALEX1931. Table 2 lists the observation date and HIRES instrumental setup. Data were reduced using the MAKEE software package which outputs heliocentric velocity-corrected spectra shifted to vacuum wavelengths. After reduction and extraction, high-order polynomials are fit to each order to bring overlapping order segments into agreement before combining both HIRES exposures and all orders. Representative HIRES data are shown in Figure 4.

\subsection{FIRE Near-infrared Spectroscopy}

Near-infrared spectroscopy was obtained with the Foldedport Infrared Echellete (FIRE; Simcoe et al. 2008, 2010) mounted on the Magellan $6.5 \mathrm{~m}$ Baade telescope. Observation parameters are listed in Table 2.

Prism-mode observations were performed with a 0.'6 slit aligned with the parallactic angle. One ABBA nod pattern was obtained for GALEX1931. A single nod pair of $1 \mathrm{~s}$ integration per nod was obtained for the telluric calibrator star HD 189920 (A0V). HD 189920 was too bright to be observed directly through the slit. The telescope was offset by $6^{\prime \prime}$ from HD 189920 and defocused until unsaturated spectra could be obtained. Although such an observation strategy would prevent accurate absolute flux calibration, the relative flux calibration should not be impaired. Data reduction followed Burgasser et al. (2010) with the exception of the dispersion solution being fit with an eighth-order Legendre polynomial (essentially equivalent to the fifth-order cubic spline employed by Burgasser et al. 2010). Absolute flux calibration of the GALEX1931 prism data is accomplished by scaling its spectrum to the ISAAC $J_{s} H K_{s^{-}}$ band measurements. The FIRE prism data are presented in Figure 3.

\section{RESULTS AND MODELING}

\subsection{Optical Spectroscopy}

Parameters for GALEX1931 are derived from model fits to all Balmer lines in the blue-side KAST data (Figure 2). 

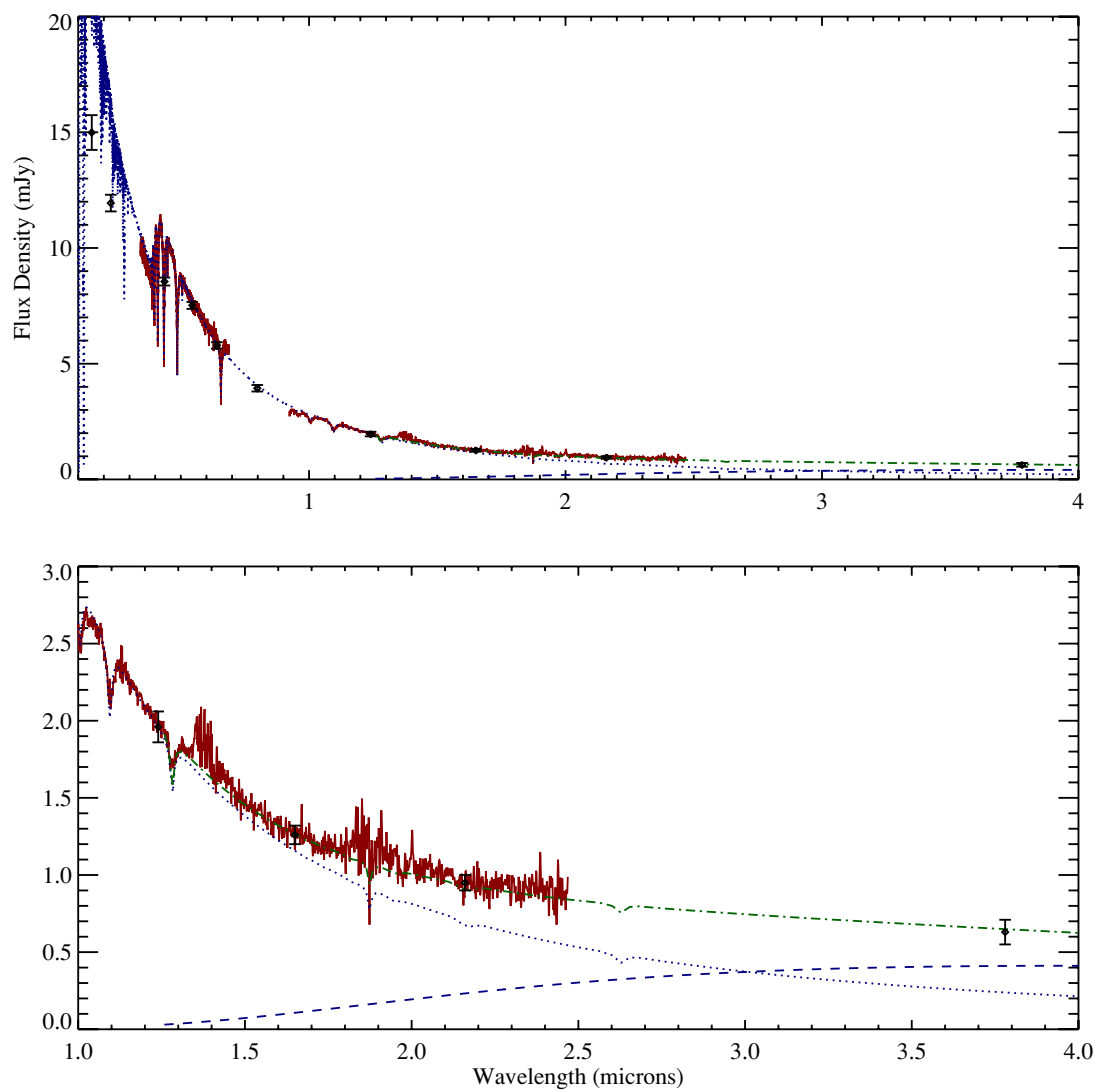

Figure 3. Spectral energy distribution for the DAZ white dwarf GALEX1931. Overplotted are models for an atmosphere of a $T_{\text {eff }}=23,500 \mathrm{~K}, \log (g)=8.0, \mathrm{DAZ}$ white dwarf with an orbiting flat, passive, opaque dust disk having $T_{\text {inner }}=1400 \mathrm{~K}, T_{\text {outer }}=1200 \mathrm{~K}$, and $i=70^{\circ}$. Data points are $G A L E X$ NUV and FUV (Section 3.1 discusses the discrepancy between the model and the GALEX NUV and FUV measurements), Nickel $B R I$, the Vennes et al. (2010) $V$, and ISAAC $J_{s} H K_{s} L^{\prime}$. The red overplotted curve in the optical is the KAST spectrum. The red overplotted curve in the infrared is the FIRE prism-mode spectrum. Both the KAST and FIRE data are flux calibrated to the broadband photometry. The blue dotted curve is the white dwarf atmospheric model and the blue dashed curve is the disk model. The sum of the two models is shown with the green dash-dotted line.

(A color version of this figure is available in the online journal.)

The method relies on the "spectroscopic technique" developed by Bergeron et al. (1992) and described at length in Liebert et al. (2005), and references therein. We find that the KAST data are best reproduced by a white dwarf with parameters $T_{\text {eff }}=23470 \pm 300 \mathrm{~K}$ and $\log g=7.99 \pm 0.05$ where $g$ has units of $\mathrm{cm} \mathrm{s}^{-2}$. We note that GALEX1931's effective temperature obtained from our fitting method differs significantly from that reported in Vennes et al. (2010) $-T_{\text {eff }}=20890 \pm 120 \mathrm{~K}$; the origin of this discrepancy is not clear at this time. By matching a model white dwarf atmosphere with our derived parameters to the optical spectra and photometry we derive a distance to GALEX1931 of $56 \pm 3 \mathrm{pc}$ - this agrees within the quoted uncertainties with the value calculated by Vennes et al. (2010). It is noted that the spectra and photometry also favor a hotter $T_{\text {eff }}$ for GALEX1931 than the value reported in Vennes et al. (2010). Cooling models similar to those described in Fontaine et al. (2001), except with carbon-oxygen cores, are used to estimate a cooling age of $30 \mathrm{Myr}$. The white dwarf mass and envelope mass are estimated to be $M_{\mathrm{WD}}=0.63 M_{\odot}$ and $\log q=-16.1$ (where $q=M_{\text {env }} / M_{\mathrm{WD}}$; Koester 2009; D. Koester 2010, private communication).

With these parameters for GALEX1931 we proceed in fitting the metallic absorption lines detected in the HIRES spectrum. We use a local thermodynamic equilibrium (LTE) model atmosphere code similar to that described in Dufour et al. $(2005,2007)$. Absorption line data are taken from the
Vienna Atomic Line Database. ${ }^{7}$ Using the effective temperature and surface gravity determined from fitting the Balmer lines, we calculate grids of synthetic spectra for each element of interest. The grids cover a range of abundances typically from $\log [n(\mathrm{Z}) / n(\mathrm{H})]=-3.0$ to -7.0 in steps of $0.5 \mathrm{dex}$. We then determine the abundance of each element by fitting the various observed lines using a similar method to that described in Dufour et al. (2005). Briefly, this is done by minimizing the value of $\chi^{2}$ which is taken to be the sum of the difference between the normalized observed and model fluxes over the frequency range of interest with all frequency points being given an equal weight. This is done individually for each line and the final adopted abundances (see Table 3 ) are taken to be the average of all the measurements made for a given element after removing outliers. Uncertainties are taken to be the dispersion among abundance values used in the average. If this dispersion is less than $0.10 \mathrm{dex}$, then the abundance uncertainty is set to 0.10 dex.

As in Vennes et al. (2010), we find contributions from Ca, $\mathrm{Mg}, \mathrm{Si}$, and $\mathrm{Fe}$. The HIRES spectra do not extend sufficiently into the red to confirm the presence of $\mathrm{O}$, so we use the Vennes et al. (2010) O I $\lambda 7777$ triplet equivalent widths to estimate the $\mathrm{O}$ abundance. We make synthetic spectra for the $\mathrm{O}$ I triplet in the same way as for the other elements. The equivalent widths

\footnotetext{
7 http://vald.astro.univie.ac.at/ vald/php/vald.php
} 

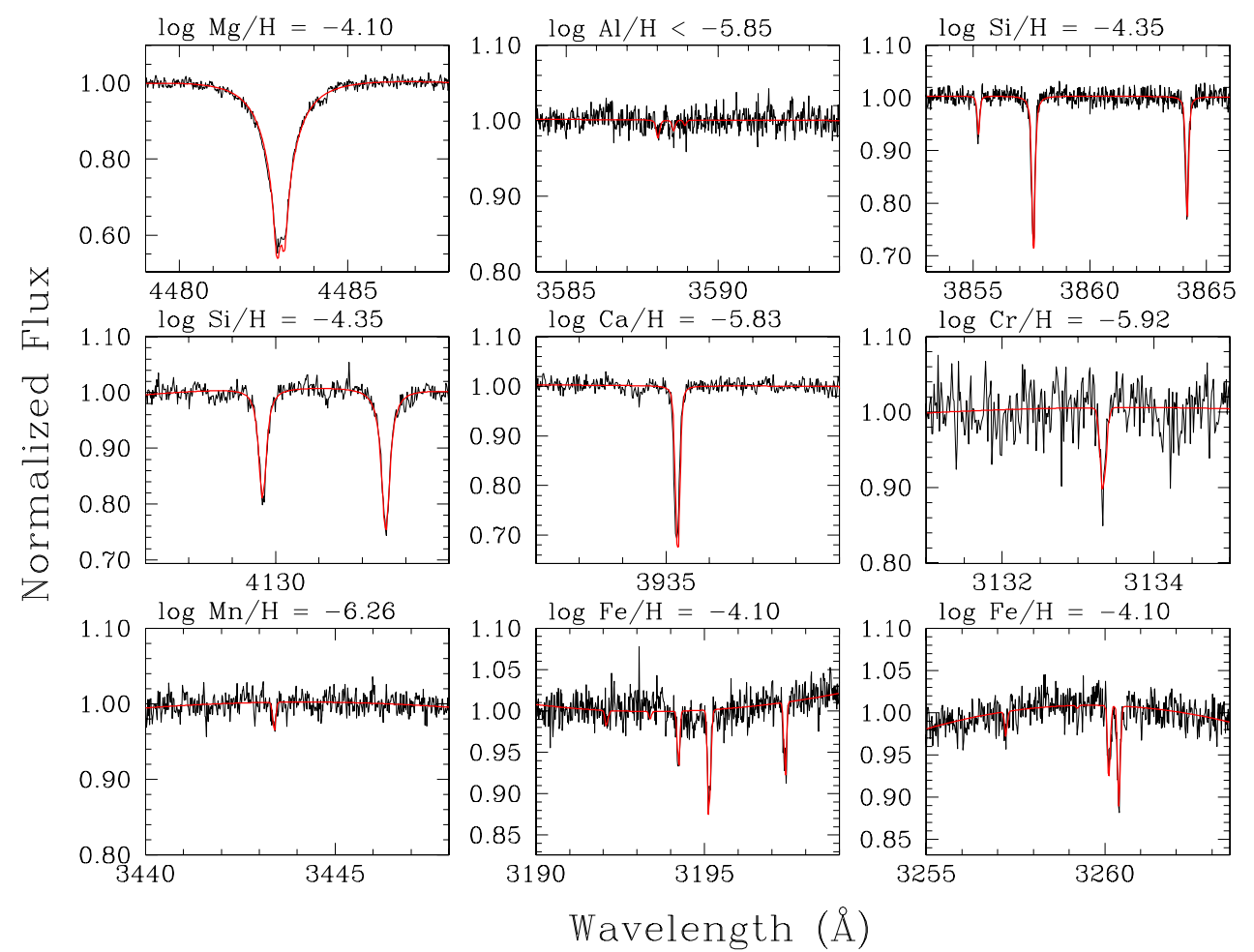

Figure 4. Elements identified in the HIRES spectra of GALEX1931. Aluminum is tentatively identified, and the indicated abundance is suggested as an upper limit. Higher S/N spectra in this region or ultraviolet spectra could confirm the detection of Al. Wavelengths are in the vacuum heliocentric reference frame. Smooth, red curves are model fits with the indicated abundances by number.

(A color version of this figure is available in the online journal.)

Table 3

GALEX1931+0117 Metal Pollution

\begin{tabular}{lccccc}
\hline \hline$Z$ & $\log [n(Z) / n(\mathrm{H})]_{\text {measured }}$ & $\tau_{\text {diff }}(\text { days })^{\mathrm{a}}$ & {$[n(Z) / n(\mathrm{Fe})]_{\text {accreted }} \mathrm{b}$} & {$[n(Z) / n(\mathrm{Fe})]_{\mathrm{CI}}{ }^{\mathrm{c}}$} & $\dot{M}_{\text {acc }} /\left(10^{7} \mathrm{~g} \mathrm{~s}^{-1}\right)^{\mathrm{d}}$ \\
\hline $\mathrm{C}$ & $<-4.85$ & 5.811 & $<0.0712$ & 0.8912 & $<3.15$ \\
$\mathrm{O}$ & $-3.68 \pm 0.10^{\mathrm{e}}$ & 3.534 & 1.7338 & 8.7096 & 102.0 \\
$\mathrm{Mg}$ & $-4.10 \pm 0.10$ & 4.532 & 0.5140 & 1.2022 & 46.1 \\
$\mathrm{Al}$ & $<-5.85$ & 5.336 & $<0.0077$ & 0.0954 & $<0.772$ \\
$\mathrm{Si}$ & $-4.35 \pm 0.11$ & 4.542 & 0.2884 & 1.1481 & 29.8 \\
$\mathrm{Ca}$ & $-5.83 \pm 0.10$ & 2.730 & 0.0158 & 0.0691 & 2.34 \\
$\mathrm{Ti}$ & $<-7.00$ & 2.960 & $<0.0010$ & 0.0027 & $<0.174$ \\
$\mathrm{Cr}$ & $-5.92 \pm 0.14$ & 2.560 & 0.0137 & 0.0151 & 2.55 \\
$\mathrm{Mn}$ & $-6.26 \pm 0.15$ & 2.292 & 0.0070 & 0.0104 & 2.64 \\
$\mathrm{Fe}$ & $-4.10 \pm 0.10$ & 2.329 & 1.00 & 1.00 & 205.0 \\
$\mathrm{Ni}$ & $<-5.60$ & 1.982 & $<0.0371$ & 0.0549 & $<8.04$ \\
\hline
\end{tabular}

Notes.

${ }^{a}$ Diffusion constants; see Koester (2009) and Section 4.

${ }^{\mathrm{b}}$ Parent body abundances relative to Fe; see Section 4 and Figure 6.

${ }^{\mathrm{c}}$ CI chondrite data from Lodders (2003). See also Figure 6.

d $\dot{M}_{\text {acc }}(Z)=M_{\text {env }}(Z) / \tau_{\text {diff }}(Z)$, where $M_{\text {env }}(Z)$ is the mass of element $Z$ in GALEX1931's envelope assuming the hydrogen-dominated envelope mass is $9.4 \times 10^{16} \mathrm{~g}$ (Koester 2009; D. Koester 2010, private communication).

e The $\mathrm{O}$ abundance is derived from the equivalent widths reported in Vennes et al. (2010); see Section 3.1.

of the synthetic $\mathrm{O}$ I lines are then compared to the Vennes et al. (2010) measured values to derive the abundance reported in Table 3. Abundance measurements for $\mathrm{Ca}, \mathrm{Si}$, and $\mathrm{O}$ are in agreement with those reported in Vennes et al. $(2010,2011)$, not withstanding the significant $T_{\text {eff }}$ difference between our white dwarf parameters and theirs. The revised $\mathrm{Mg}$ abundance quoted in Vennes et al. (2011) is less abundant by $\approx 0.3$ dex due entirely to the Vennes et al. (2011) inclusion of pressure broadening and non-LTE effects. We find an Fe abundance higher than Vennes et al. $(2010,2011)$ by $\approx 0.4$ dex. It is noted that fits to the Fe lines assuming a $T_{\text {eff }}$ of $21,000 \mathrm{~K}$ yield an Fe abundance consistent with that reported in Vennes et al. (2010, 2011). New elements discovered in the HIRES data are chromium identified from two $\mathrm{Cr}$ II lines and manganese identified from one Mn II line (Figure 4). Aluminum may be present in the HIRES data as well (Figure 4); however, the signal-to-noise ratio $(\mathrm{S} / \mathrm{N})$ of the detection is marginal so we report $\mathrm{Al}$ only as an upper limit (Table 3). Abundances for all elements identified to date in the atmosphere of GALEX1931 are reported in Table 3 as well as upper limits for other elements of interest (see Section 4).

It is noted that the GALEX NUV and FUV measurements appear discrepant with the white dwarf parameters derived 


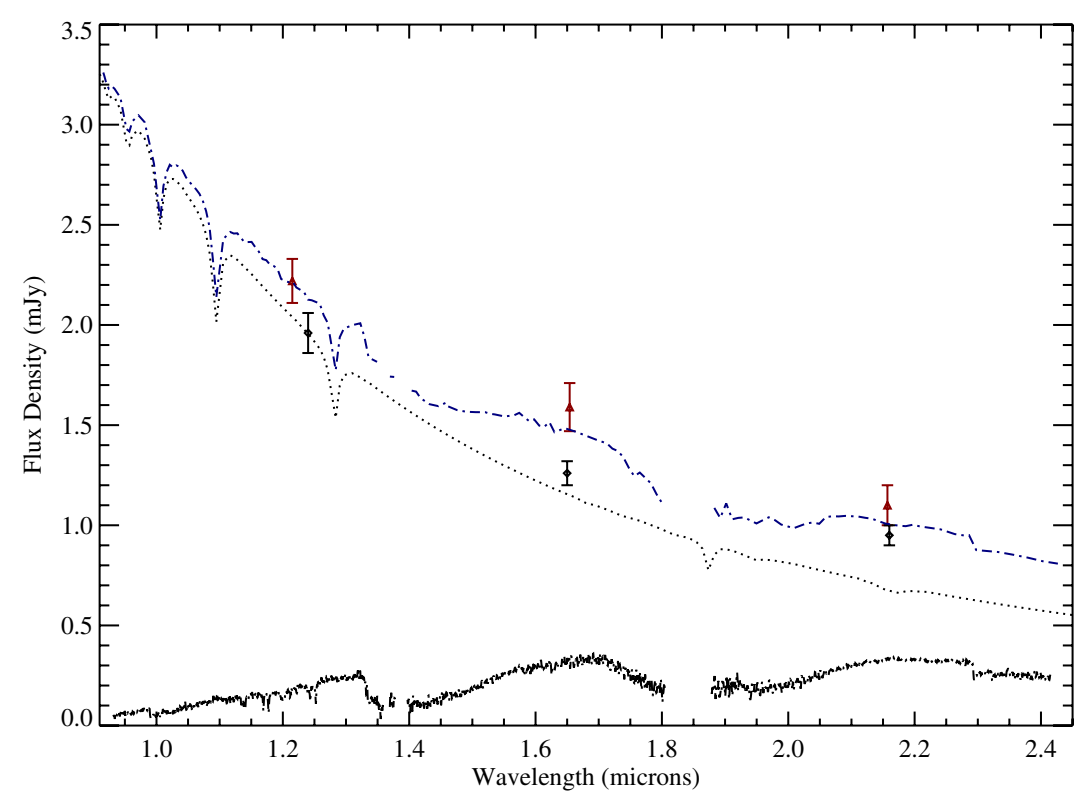

Figure 5. Comparison of near-infrared measurements to models for a mid-L-type brown dwarf orbiting GALEX1931. The red, upper data points are the 2MASS photometry; the black, lower data points are the ISAAC photometry. The black dotted curve at the bottom of the plot is mid-L-type brown dwarf spectral data from the SpeX spectral library (Cushing et al. 2005; Rayner et al. 2009) shifted in flux to the distance estimated for GALEX1931 (Section 3.1). The dotted curve in the middle of the plot is the atmospheric model for GALEX1931 shown in Figure 3. The blue dash-dotted curve is the sum of the white dwarf and brown dwarf curves for an L5 type brown dwarf. The FIRE data smoothly connect the ISAAC data points and show no features.

(A color version of this figure is available in the online journal.)

above. We find that the model can be brought into agreement with the data by applying the Cardelli et al. (1989) extinction curve assuming $E(B-V)=0.03$ and interstellar reddening. However, such reddening places the model at the limit of the $3 \sigma$ uncertainties quoted for the optical photometry. It is likely that the true color excess toward GALEX1931 is less than 0.03 and that some combination of reddening and metal line blanketing (from elements detected and as of yet undetected) in the UVspectrum of GALEX1931 are responsible for the depressed GALEX measurements relative to the model.

\subsection{Infrared Excess Modeling}

Table 1 lists measured ultraviolet, optical, and near-infrared fluxes for GALEX1931. These data are presented with the KAST and FIRE prism-mode data in Figure 3. Comparison of Table $1 J_{s} H K_{s}$ magnitudes to the 2MASS magnitudes $\left(J=14.66 \pm 0.05, H=14.55 \pm 0.09, K_{s}=14.45 \pm 0.10\right.$; Skrutskie et al. 2006) suggests that the 2MASS measurements for GALEX1931 suffer from contamination in the photometric apertures and/or annuli used to calculate the sky background and hence are inaccurate. One can identify at least two background sources in Figure 1 that may have fallen within the 2MASS apertures in this crowded field near the Galactic plane. Similarly, the WISE data reported in Debes et al. (2011) could have been contaminated by these same sources. Due to the discrepancy between the WISE data and the higher angular resolution data presented herein, we do not include WISE data in our analysis. The ISAAC $J_{s^{-}}$and $H$-band measurements agree within errors with the white dwarf photospheric model, while the $K_{s}$-band measurement confirms the upward slope of the FIRE spectrum demonstrating excess emission that begins at wavelengths near $2 \mu \mathrm{m}$.

Figure 5 illustrates that the excess emission implied by the contaminated 2MASS photometry is potentially consistent with an L-type companion; this fact motivated Vennes et al.
(2010) to consider a model where GALEX1931 is orbited by a mid-L-type companion. The ISAAC photometry, which is of higher fidelity, clearly indicates that a mid-L-type object cannot be the cause of the excess infrared emission. Later type companions are ruled out by the ISAAC photometry (e.g., Farihi et al. 2005) and FIRE spectra: the spectra smoothly connect the ISAAC $J_{s} H K_{s}$ data points and show no evidence for the expected spectral features arising from a sub-stellar companion (Farihi \& Christopher 2004).

Given the volume of previous results linking white dwarf metal pollution and dusty disks (e.g., Farihi et al. 2009), it seems reasonable to conclude that the observed excess infrared emission detected toward GALEX1931 emanates from a disk that orbits (and is accreted by) the white dwarf star. A model for the photospheric emission of GALEX1931 is computed with the stellar parameters described in Section 3.1 and the detected metals with abundances as listed in Table 3. To fit the observed near-infrared spectra and photometry, we add to the white dwarf model an optically thick, flat dust disk (Jura 2003; Jura et al. $2007 \mathrm{a})$ with an inner disk temperature $\left(T_{\text {inner }}\right)$ of $1400 \mathrm{~K}$, outer disk temperature $\left(T_{\text {outer }}\right)$ of $1200 \mathrm{~K}$, and an inclination angle $(i)$ of $70^{\circ}$ (where $i=90^{\circ}$ is edge on to our line of sight). In this fit, we set $R_{\mathrm{WD}} / D=5.3 \times 10^{-12}$ (see Section 3.1). This disk and star model is shown in Figure 3 and reproduces well the observed data. It is noted that the fit presented here is not unique and that the temperature of the outer region of GALEX1931's disk is not robustly constrained by the present data.

For the case of optically thick, flat dust disks Equation (1) from Jura (2003) can be used to determine at what radial distance from a white dwarf dust particles of certain temperatures reside; for GALEX1931 $T_{\text {inner }}$ of $1400 \mathrm{~K}$ suggests particles as close as $25 R_{*}$. A temperature of $1400 \mathrm{~K}$ is close to the sublimation temperature for some silicates, an expected mineralogical constituent of GALEX1931's dusty disk given the stoichiometry of other white dwarf disks (Jura et al. 2009a; Reach et al. 2009) and the metals detected in its atmosphere (Table 3). Thus, the 
modeled inner disk radius could be physically motivated by sublimation of grains that will eventually be accreted by GALEX1931. Our data set is not comprehensive enough to rule out a warped disk morphology which could increase the inner disk radius while still reproducing the observed near-infrared excess emission (e.g., Jura et al. 2007b, 2009a, and references therein). It is noted that if a flat dust disk geometry accurately describes the distribution of GALEX1931's orbiting debris, then the disk is likely a narrow ring as flat dust disk models with $T_{\text {outer }} \leqslant$ $1000 \mathrm{~K}\left(R_{\text {outer }} \geqslant 40 R_{\mathrm{WD}}\right)$ are not consistent with the data. Such a result suggests that GALEX1931 may host a narrow distribution of disk material (e.g., see Farihi et al. 2010b).

In their red echelle spectra of GALEX1931, Vennes et al. (2010) do not identify double-peaked emission lines from the Ca II infrared triplet indicative of an orbiting gaseous disk (Gänsicke et al. 2006; Melis et al. 2010). Modeling and results presented by Melis et al. (2010) and Jura (2008) suggest that gas disks around white dwarfs are fed by an influx of rocky objects into the Roche limit of a white dwarf star. The absence of such emission lines, and hence an obvious gas disk orbiting GALEX1931, is suggestive of its dust disk originating in the tidal shredding of one large rocky object.

\section{DISCUSSION}

Modeling of the ultraviolet through infrared spectral energy distribution of GALEX1931 provides strong evidence that an orbiting dusty circumstellar disk emits the detected excess infrared emission. Following earlier results, we assume that this dusty disk has its origin in the tidal disruption of a rocky object that breached GALEX1931's Roche limit. The ultimate fate of such material is to be accreted by the white dwarf star. Under this assumption, we can deduce the bulk elemental composition of the disrupted object from the metal abundances reported in Table 3. To properly convert the measured abundances to the abundances at the time of accretion, we must include the effects of diffusion, radiative levitation, and the relevant accretion phase (Koester 2009; Chayer \& Dupuis 2010; Jura et al. 2009b, and references therein). The presence of a disk orbiting GALEX1931 and the extremely short elemental diffusion constants (Table 3) implies that we are observing elemental abundances during the steady-state accretion phase. In this phase, accretion of metals has reached an equilibrium with the rate at which these metals diffuse out of the white dwarf's photosphere. The work of Chayer \& Dupuis (2010) suggests that for accretion rates $\gtrsim 10^{4} \mathrm{~g} \mathrm{~s}^{-1}$ the effects of radiative levitation are negligible-all measured accretion rates and upper limits exceed this threshold value (Table 3 ) and hence we ignore radiative levitation. Thus, we determine the accreted abundance ratios by employing Equation (16) from Jura et al. (2009b) and the diffusion constants of Koester (2009) computed specifically for the GALEX1931 parameters determined in Section 3.1 (D. Koester 2010, private communication). Accreted abundances relative to Fe are reported in Table 3.

We desire to make a rough estimate of the mass accreted to date by GALEX1931. Accretion rates for each element are computed under the assumption that material accretes in the steady-state; these values are reported in the right-hand column of Table 3. The total accretion rate summed over all elements is $\sim 3.9 \times 10^{9} \mathrm{~g} \mathrm{~s}^{-1}$. An estimate of how long GALEX1931 has been accreting from its dusty disk comes from the percentage of DA stars with effective temperatures similar to GALEX1931 and atmospheric pollution. It is assumed that this percentage is linked to the length of time in which such DA white dwarf stars would show metal pollution and hence be polluted from a surrounding disk of material. From Koester et al. (2005, 2009), we find that 5 out of 116 or $4.31 \%$ of DA white dwarfs within the temperature range of $19,500 \mathrm{~K}<T_{\text {eff }}<25,000 \mathrm{~K}$ are polluted with metals (including GALEX1931). Taking the cooling time of a white dwarf in the above $T_{\text {eff }}$ range to be equal to that of GALEX1931 (30 Myr, see Section 3.1), and assuming all white dwarfs accrete from a disk during this interval, it is determined that the disk lifetime for GALEX1931 is 1.3 Myr. Assuming GALEX1931 has been accreting at a constant rate for $\sim 1 \mathrm{Myr}$, $\sim 2 \times 10^{23} \mathrm{~g}$ of material has been accreted by the white dwarf star. It is noted that numerous assumptions are included in this estimate. The mass of the second most massive solar system asteroid, Vesta, is $\approx 3 \times 10^{23} \mathrm{~g}$.

Following Klein et al. (2010), we attempt to account for the accreted oxygen under the expectation that all oxygen was contained in rocky oxygenated minerals (e.g., $\mathrm{MgO}, \mathrm{Al}_{2} \mathrm{O}_{3}$, $\mathrm{SiO}_{2}, \mathrm{CaO}, \mathrm{TiO}_{2}, \mathrm{Cr}_{2} \mathrm{O}_{3}, \mathrm{MnO}, \mathrm{FeO}, \mathrm{Fe}_{2} \mathrm{O}_{3}$, and $\mathrm{NiO}$ ) and water $\left(\mathrm{H}_{2} \mathrm{O}\right.$; see Jura \& Xu 2010 for a discussion of water survival in rocky objects during post-main sequence evolution). Inherent to such balancing is the assumption that the tidally shredded remnants of the parent body are well mixed in the disk. The criterion that needs to be met is Equation (3) of Klein et al. (2010):

$$
O_{\mathrm{bal}} \equiv \sum_{Z} \frac{q(Z)}{p(Z)} \frac{n(Z)}{n(\mathrm{O})}=1
$$

where element $Z$ is contained in molecule $Z_{q(Z)} \mathrm{O}_{p(Z)}$. In this equation, one must consider only hydrogen accreted from the parent body $\left(H_{\mathrm{acc}}\right)$. We find that the oxygen being accreted by GALEX1931 can be accounted for almost entirely by inclusion in rocky minerals. There is, however, the ambiguity of how Fe was partitioned in the parent body that was accreted by GALEX1931. In our model, Fe could have been contained in any one of metallic $\mathrm{Fe}, \mathrm{FeO}$, or $\mathrm{Fe}_{2} \mathrm{O}_{3}$ (see discussion in Klein et al. 2010). Depending on how we divide Fe into these three states, there can be anything from a significant deficit in the oxygen balance ( $O_{\text {bal }}=0.58$ instead of 1.0 , indicating too much oxygen for the number of heavier element atoms) to a significant overestimate of rocky minerals $\left(O_{\mathrm{bal}}=1.33\right.$ instead of 1.0, indicating too little oxygen for the number of heavier element atoms). Assuming as did Klein et al. (2010) that half of the Fe is in the form of metallic $\mathrm{Fe}$ and half in the form of $\mathrm{FeO}$, we arrive at a value of $O_{\text {bal }}=0.83$. With this value we constrain the amount of water present in the object being accreted by GALEX1931 to be $<1 \%$ of the mass of the parent body. It is noted that use of the lower $\mathrm{Mg}$ abundance derived by Vennes et al. (2011) would result in a more water-rich body. Thus, it appears as though the material accreting onto GALEX1931 is very likely to have originated in rocky minerals and is likely to be only a small percentage of water by mass.

Examination of the bulk elemental composition of the material that is accreted by GALEX1931 can potentially yield insight into the evolutionary processes that affect terrestrial-like planets during the late stages of stellar evolution. In Figure 6, we plot the elemental abundances relative to Fe for three white dwarfs: GD 362 (Zuckerman et al. 2007; Koester 2009), GD 40 (Klein et al. 2010), and GALEX1931. We normalize each of these relative abundance measurements (at the time of accretion for each 


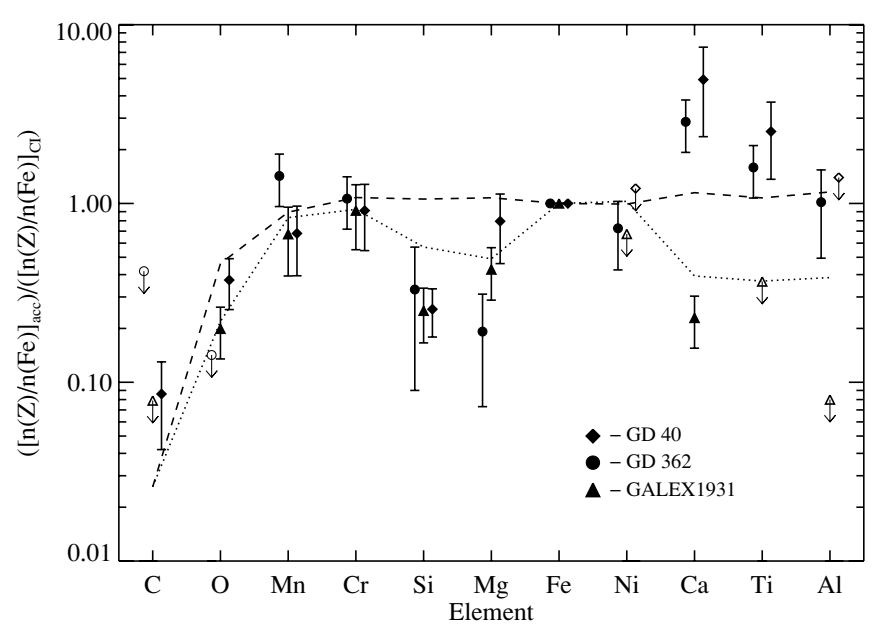

Figure 6. Comparison of white dwarf abundances relative to $\mathrm{Fe}$ to $\mathrm{CI}$ chondrite abundances relative to Fe (after Figure 15 of Klein et al. 2010). Elements have increasing condensation temperature to the right. The triangle data points are measurements and upper limits for GALEX1931 from Table 3. The diamond data points are measurements and upper limits for GD 40 (Klein et al. 2010), while the circle data points are measurements and upper limits for GD 362 (Zuckerman et al. 2007; Koester 2009). The dashed line is data for the bulk Earth (Allègre et al. 1995; van Thienen et al. 2007). The dotted line is a "windstripped Earth" model as described in Section 4.

object) to elemental abundances relative to Fe for CI chondrites (see Table 3 and Lodders 2003). CI chondrites are chosen as these objects have some of the best-determined bulk compositions of any object in our solar system. CI chondrites have nearsolar compositions and thus represent primitive rocks that likely formed directly from the solar nebula (e.g., Lodders 2003 and references therein). Under the assumption that primitive rocks are similar in all planetary systems, we seek to probe whether or not the parent body being accreted by GALEX1931 has undergone significant processing by comparing the GALEX1931 measured abundances to CI chondrite abundances. To further place the white dwarf measurements into context, we overplot the bulk elemental composition of Earth as extracted from the measurements of Allègre et al. (1995) and the compilation of van Thienen et al. (2007).

Most striking in Figure 6 is the agreement of the abundances of some elements for the three white dwarfs with the bulk Earth, especially the depletion of the volatiles $\mathrm{C}$ and $\mathrm{O}$ relative to what would be expected from the more primitive CI chondrites. Next is the good agreement between transition metal abundances ( $\mathrm{Mn}, \mathrm{Cr}, \mathrm{Fe}$, and $\mathrm{Ni}$ ) for the accreted objects and the bulk Earth. The obvious discrepancies are with $\mathrm{Si}$ and $\mathrm{Mg}$ and the refractories $\mathrm{Ca}$ and $\mathrm{Ti}$. Assuming the parent bodies that are accreted by each white dwarf started close to Earth-like in bulk elemental composition (the best assumption that can be made at present, but see the encouraging work of Bond et al. 2010), significant deficits of $\mathrm{Si}$ and varying degrees of deficient $\mathrm{Mg}$ suggest crustal and mantle loss. This result suggests that each white dwarf has accreted objects massive enough to experience differentiation. That is, the atoms layered themselves in the parent body by mass (with the heaviest atoms at the parent body's core). Such a result indicates that the parent bodies now being accreted by each white dwarf were indeed massive bodies and perhaps even full-fledged planets.

$\mathrm{Ca}, \mathrm{Ti}$, and $\mathrm{Al}$ abundances could potentially bear the signature of a particular crust and mantle loss mechanism. Klein et al. (2010) compared the accreted abundances for GD 40 to a model developed by Fegley \& Cameron (1987) that described the evolution of a rocky planetary body when it is exposed to intense radiation. The predictions of this model are in good agreement with the parent body abundances for the object accreted by GD 40 (Klein et al. 2010). Such a model could potentially provide a reasonable explanation for the parent body composition of the object accreted by GD 362 as it too shows the characteristic enhancement of $\mathrm{Ca}$ and $\mathrm{Ti}$ predicted in the Fegley \& Cameron (1987) model (Figure 6). To explain the results for GALEX1931 requires either additional evolutionary processes or a different evolutionary path that results in crust and mantle loss without the enhancement of $\mathrm{Ca}$ and Ti. Indeed, GALEX1931 shows a significant deficit of $\mathrm{Ca}, \mathrm{Ti}$, and $\mathrm{Al}$ relative to the Earth and $\mathrm{CI}$ chondrites (see Figure 6).

To explain the distinctive elemental mixture being accreted by GALEX1931, we propose a scenario in which the dominant evolutionary mechanism acting on the parent body was interaction with the host star stellar wind. After irradiation by the luminous host star while on the asymptotic giant branch (AGB), interaction with the AGB ejecta seems a likely choice for a rocky planet mass loss mechanism (e.g., Jura 2008). In such a scenario we envision that the rocky planet is essentially sanded down by the AGB wind, losing first its crust then mantle depending on the severity of the planet's interaction with the stellar wind. We estimate to first order the relative abundances of such an object after crustal and mantle stripping. We begin with the assumption that the object started with the same composition as the bulk Earth and the same crust and mantle abundances as the Earth (Anderson 1989; Allègre et al. 1995). We remove varying fractions of the crust, then upper mantle, and then lower mantle. After this removal step, the bulk abundances of the object are recomputed relative to Fe. Experimentation with varying degrees of crust and mantle removal suggests that the best match between the simple stripping model and the GALEX1931 abundances shown in Figure 6 is obtained after stripping the entire crust and upper mantle and half of the lower mantle. The simple model provides good agreement with the measurements, with the exception of aluminum. These results suggest that "wind stripping" of the outer layers of an Earth-like planet is a viable mode to produce the observed parent body abundances in at least some white dwarfs and thus that interaction with the AGB stellar wind could be an important process in the final evolutionary phase of a terrestrial-like planet. Exploration of the relevant physics in the wind stripping process can further develop this model.

\section{CONCLUSIONS}

We have carried out a suite of observations aimed at characterizing GALEX1931's infrared excess and atmospheric pollution. Our broad data set allows us to identify the excess infrared emission detected toward GALEX1931 as emanating from an orbiting dusty debris disk. Analysis of the heavy metals detected in GALEX1931's atmosphere suggests that it is accreting the remnants of a differentiated - and hence massive_rocky body. Assuming the object being accreted by GALEX1931 started out roughly Earth-like in composition, comparison of the distinctive bulk elemental composition of this terrestrial-like planet to predictions from a simple wind-stripping model suggests that the planet's outer layers were removed before the remaining portion of the planet was accreted by the white dwarf star. A likely culprit for such stripping is interaction between the planet and its host star's dense stellar wind while the star progresses through the AGB. 
C.M. was supported by the National Science Foundation under award No. AST-1003318. P.D. is a CRAQ postdoctoral fellow. We thank Detlev Koester for kindly providing diffusion constants tailored to GALEX1931. Some of the data presented herein were obtained at the W. M. Keck Observatory, which is operated as a scientific partnership among the California Institute of Technology, the University of California, and the National Aeronautics and Space Administration. The Observatory was made possible by the generous financial support of the W. M. Keck Foundation. This paper includes data gathered with the $6.5 \mathrm{~m}$ Magellan Telescopes located at Las Campanas Observatory, Chile. This publication makes use of data products from the Two Micron All Sky Survey, which is a joint project of the University of Massachusetts and the Infrared Processing and Analysis Center/California Institute of Technology, funded by the National Aeronautics and Space Administration and the National Science Foundation. This research has made use of the SIMBAD database. Based on observations made with the NASA Galaxy Evolution Explorer. GALEX is operated for NASA by the California Institute of Technology under NASA contract NAS5-98034. This research was supported in part by NASA grants to UCLA. This work is supported in part by the NSERC Canada and by the Fund FQRNT (Québec).

Facilities: Keck:I (HIRES), VLT:Melipal (ISAAC), Magellan:Baade (FIRE), Nickel (Direct Imaging Camera), Shane (KAST)

\section{REFERENCES}

Allègre, C. J., Poirier, J., Humler, E., \& Hofmann, A. W. 1995, Earth Planet. Sci. Lett., 134, 515

Anderson, D. L. 1989, Theory of the Earth (Oxford: Blackwell)

Bergeron, P., Saffer, R. A., \& Liebert, J. 1992, ApJ, 394, 228

Bessell, M. S. 1990, PASP, 102, 1181

Bond, J. C., O’Brien, D. P., \& Lauretta, D. S. 2010, ApJ, 715, 1050

Burgasser, A. J., et al. 2010, ApJ, 725, 1405

Cardelli, J. A., Clayton, G. C., \& Mathis, J. S. 1989, ApJ, 345, 245

Chayer, P., \& Dupuis, J. 2010, in AIP Conf. Proc. 1273, 17th European White Dwarf Workshop, ed. K. Werner \& T. Rauch (Melville, NY: AIP), 394

Cushing, M. C., Rayner, J. T., \& Vacca, W. D. 2005, ApJ, 623, 1115

Debes, J. H., et al. 2011, ApJ, 724, 4

Dufour, P., Bergeron, P., \& Fontaine, G. 2005, ApJ, 627, 404

Dufour, P., Kilic, M., Fontaine, G., Bergeron, P., Lachapelle, F., Kleinman, S. J., \& Leggett, S. K. 2010, ApJ, 719, 803
Dufour, P., et al. 2007, ApJ, 663, 1291

Farihi, J., Barstow, M. A., Redfield, S., Dufour, P., \& Hambly, N. C. 2010a, MNRAS, 404, 2123

Farihi, J., Becklin, E. E., \& Zuckerman, B. 2005, ApJS, 161, 394

Farihi, J., \& Christopher, M. 2004, AJ, 128, 1868

Farihi, J., Jura, M., Lee, J., \& Zuckerman, B. 2010b, ApJ, 714, 1386

Farihi, J., Jura, M., \& Zuckerman, B. 2009, ApJ, 694, 805

Fegley, B., \& Cameron, A. G. W. 1987, Earth Planet. Sci. Lett., 82, 207

Fontaine, G., Brassard, P., \& Bergeron, P. 2001, PASP, 113, 409

Gänsicke, B. T., Marsh, T. R., Southworth, J., \& Rebassa-Mansergas, A. 2006, Science, 314, 1908

Hawarden, T. G., Leggett, S. K., Letawsky, M. B., Ballantyne, D. R., \& Casali, M. M. 2001, MNRAS, 325, 563

Jura, M. 2003, ApJ, 584, L91

Jura, M. 2008, AJ, 135, 1785

Jura, M., Farihi, J., \& Zuckerman, B. 2007a, ApJ, 663, 1285

Jura, M., Farihi, J., \& Zuckerman, B. 2009a, AJ, 137, 3191

Jura, M., Farihi, J., Zuckerman, B., \& Becklin, E. E. 2007b, AJ, 133, 1927

Jura, M., Muno, M. P., Farihi, J., \& Zuckerman, B. 2009b, ApJ, 699, 1473

Jura, M., \& Xu, S. 2010, AJ, 140, 1129

Klein, B., Jura, M., Koester, D., Zuckerman, B., \& Melis, C. 2010, ApJ, 709, 950

Koester, D. 2009, A\&A, 498, 517

Koester, D., Rollenhagen, K., Napiwotzki, R., Voss, B., Christlieb, N., Homeier, D., \& Reimers, D. 2005, A\&A, 432, 1025

Koester, D., et al. 2009, A\&A, 505, 441

Landolt, A. U. 2009, AJ, 137, 4186

Leggett, S. K., et al. 2003, MNRAS, 345, 144

Leggett, S. K., et al. 2006, MNRAS, 373, 781

Liebert, J., Bergeron, P., \& Holberg, J. B. 2005, ApJS, 156, 47

Lodders, K. 2003, ApJ, 591, 1220

Martin, D. C., et al. 2005, ApJ, 619, L1

Melis, C., Jura, M., Albert, L., Klein, B., \& Zuckerman, B. 2010, ApJ, 722, 1078

Moorwood, A., et al. 1998, Messenger, 94, 7

Morrissey, P., et al. 2007, ApJS, 173, 682

Rayner, J. T., Cushing, M. C., \& Vacca, W. D. 2009, ApJS, 185, 289

Reach, W. T., Lisse, C., von Hippel, T., \& Mullally, F. 2009, ApJ, 693, 697

Simcoe, R. A., et al. 2008, Proc. SPIE, 7014, 27

Simcoe, R. A., et al. 2010, Proc. SPIE, 7735, 38

Skrutskie, M. F., et al. 2006, AJ, 131, 1163

van Thienen, P., Benzerara, K., Breuer, D., Gillmann, C., Labrosse, S., Lognonné, P., \& Spohn, T. 2007, Space Sci. Rev., 129, 167

Vennes, S., Kawka, A., \& Németh, P. 2010, MNRAS, 404, L40

Vennes, S., Kawka, A., \& Nemeth, P. 2011, arXiv:1101.2113

Vogt, S. S., et al. 1994, Proc. SPIE, 2198, 362

Zuckerman, B., Koester, D., Melis, C., Hansen, B. M., \& Jura, M. 2007, ApJ, 671,872

Zuckerman, B., Melis, C., Klein, B., Koester, D., \& Jura, M. 2010, ApJ, 722, 725 